\title{
A Special Section on Intelligent Computing and Network Security in Healthcare
}

Aneel Rahim

Technological University Dublin, aneel.rahim@tudublin.ie

Adeel Javed

University of Lahore

Follow this and additional works at: https://arrow.tudublin.ie/scschcomoth

Part of the Computer Sciences Commons

\section{Recommended Citation}

Rahim, Aneel \& Javed, Adeel. (2017). A Special Section on Intelligent Computing and Network Security in Healthcare. Journal of Medical Imaging and Health Informatics. 7. 653-654. 10.1166/jmihi.2017.2038.

This Article is brought to you for free and open access by the School of Computer Sciences at ARROW@TU Dublin. It has been accepted for inclusion in Other resources by an authorized administrator of ARROW@TU Dublin. For more information, please contact arrow.admin@tudublin.ie, aisling.coyne@tudublin.ie, gerard.connolly@tudublin.ie.

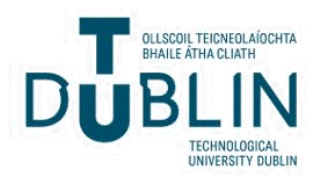




\title{
PREFACE SI on Intelligent Computing and Network Security in Healthcare
}

\author{
Aneel Rahim, Adeel Javed \\ Dublin Institute of Technology, Dublin, Ireland \\ Department of Computer Science, \\ University of Otago, New Zealand \\ aliahmed263@yahoo.com, adeel2122@gmail.com
}

\begin{abstract}
We are living in communication age and use of latest technology in the field of Medical IT Networks is offering many new services but at the same time raising various security issues. Securing the computer communication by keeping all the services available and cost effective is one of the major challenges of current healthcare applications. Mobility is something that will be the requirement of each and every type of network technology in coming few years. So mobile communication adds more in the security issues and opens many doors for the attacker to invade. Researchers all over the world are developing solution to close those doors from where attacks can be launched. In short providing security in healthcare is one of the major challenges of the current age. This Special Issue presents a total of 15 selected papers in the most active areas of network security research in healthcare.
\end{abstract}

Alshammari and Almutairi propose a utilization of knowledge discovery on healthcare data, in particular building a diabetes early warning system, to predict diabetes' risk factors that can cause that disease or increase the risk of developing diabetes in non-diabetic patients. Three data mining algorithms have been used; RIPPER, C4.5 decision tree, and AdaBoost (Meta algorithm). As a result of applying these algorithms in real diabetes data sets, a prediction model were constructed with high prediction accuracy.

Jihun Choi et. al, designed a mobile application for a daily assessment of forearm pronation and supination motor activities as an early diagnostic tool of Parkinson's disease (PD). This application uses mobile phones which collect real-time forearm pronation and supination movement activities using the phone's tri-axial accelerometer. It would be useful for early diagnosis of patients with PD and for the personalized treatment plan adjustments.

Kwang Baek Kim et. al, developed fully automatic vision based liver and kidney area extraction subsystem. Actually, this extracting region-of-interest (ROI) stage usually requires manual procedure or human intervention with computer aided tools. Thus, our vision based fully automatic approach that extracts the liver area and the kidney area separately is rare to find. In experiment with human medical experts' verification, our software was successful to extract the liver and the kidney area with $87.5 \%$ $95 \%$ in accuracy.

Keun Lee and Joon Park, created a neighborhood park information map providing information for healthcare by using UAV (Unmanned Aerial Vehicle). As a result, it was possible to effectively produce image-based information that residents can comfortably utilize and access. Information map includes the consumed calories according to types of exercise, grade and length of trail which has calculated using estimation equation of energy expenditure regarding workout hours for users' healthcare. Therefore, users' interest can be attracted and the contained information about exercise will contribute to motivation for exercise.In addition, geospatial information will be expect to use for park maintenance and design for healthcare as a basic data in the future.

Kwang Kim et al, focused on the effect of image preprocessing method with cell-specific morphological features in extracting target nucleus from images with multiple overlapped cells. The Lighting compensation method is the key of their preprocessing and other methods such as $3 \times 3$ mask noise removal and Grassfire labeling with cell specific morphological features in recognizing cancer cell, they obtained a decent extraction accuracy of $88.8 \%$ overall without any other complicated intelligent tools such as neural network and fuzzy clustering.

JinSoo Hwang and Kichang Kim suggested a systematic technique to validate the security of the imported bundle by tracing the call chain of all suspicious methods. Becuse the Previous researches on Open Service Gateway Initiative security system mainly focus on security policy enforcement or the completeness of the bundle permission file. However, permission checking at bundle level does not always guarantee the security of the system. Further, they explained the technique in detail and showed promising experimental result.

Hyeon Joeng, and Yoo Choi investigated the effect of cold-water immersion on the recovery of body fatigue recipient. This work based on a quasi-experiment study aimed to develop an effective intervention method for fatigue by analyzing the effect on fatigue level, stress index, and autonomic activity. Cold-water immersion, targeting the legs, was applied 
in university students with body fatigue induced by treadmill exercise.

Huda AlGhamdi, and Riyad Alshammari worked to classify readmitted and not-readmitted patients at King Abdul-Aziz Medical City (KAMC) in Riyadh, Saudi Arabia using a set of classifications techniques. Support Vector Machines (SVM), Neural Network (NN), C4.5 Decision Tree (DT) and Rules-Based techniques are applied on a dataset consists of admissions/readmissions events between January 2010 and April 2015. 132,788 records are the total number of dataset's instances after preprocessing. The results show that readmission within 15 days is more predictable than readmission occur within 30 days. SVM shows the highest accuracy (93.10\%).

Basma Al Saab and Bandar Alhaqbani measured the level of interoperability. Hospital Information System (HIS), Laboratory Information System (LIS), and Radiology Information System (RIS) in National Guard Health Affairs (NGHA) in Saudi Arabia, which provides insight about the current interoperability level and how to improve it. The result showed that NGHA has a high interoperability level because of their adopted integration engine that links all systems together.

Basit Shahzad et al, discussed socio-technical challenges and mitigation guidelines in developing mobile healthcare applications. They addressed the risks faced by mobile application developments, particularly eHealth applications. The paper results include a description for selecting risks and propose a mitigation strategy for overcoming development risks to ensure the construction of effective, secure, standard, and results-oriented healthcare applications beneficial to humanity.

Buthina et. al, worked on intrusion detection for mobile healthcare information systems because network security is the most important subject matter which becoming intolerable to protect the data from vulnerable attacks. Therefore, intrusion detection systems (IDS) appeared as the key solution for detecting these attacks so that the network remains reliable.

Fazal-e-Amin et al, focused on security requirements and concerns of cloud based C4I systems. Cloud computing and C4I systems are discussed from the security point of view. Furthermore, an enhanced encryption algorithm to improve data confidentiality is presented and its security is analyzed. This work can be helpful for both academia and industry to further strengthen the basis of cloud based C4I systems by improving data confidentiality.

Asrar Sabir and Ahmad Isha focused on major health care issue among heavy vehicle drivers in context of Malaysian oil and gas Industry and highlighted the importance of safety training interventions in abating fatal crashes among truck drivers. According to the Malaysian Institute of Road
Safety Research (MIROS), there are unprecedented fatalities and accidents among heavy vehicle drivers. This is a major health care issue for this industry.

Abrar et. at, conducted a comparative study of various kernels of kernel principal component analysis (KPCA) in intrusion detection that is an improvement step toward security in healthcare sector. As the KPCA has different kernel for feature transformation. Thus, need to investigate different kernels and find the best one for the intrusion detection problem, which is necessary to secure systems in healthcare. The focus of this work is to explore performance of various kernels of KPCA. Three kernels; Gaussian kernel, Polynomial kernel and Tanh kernel are investigated on standard dataset of intrusion detection. Different experiments were conducted to find the best performance of the kernels in intrusion detection.

Noor Zaman and Muneer Ahmad evaluated different authentication protocols for mobile command and control unit in healthcare because command and control systems are playing very important role in any critical situation. These systems help in situation awareness, decision making, and prompt response in emergencies. Command and control systems have been used in various organizations such as defense, civil and healthcare. Although, the command and control systems have several applications but they are also considered favorite target of hackers and intruders.

\section{Acknowledgement}

We are grateful to all authors who submitted their papers for publication in this Special Issue. We would also like to acknowledge the great efforts of the reviewers to complete this task on time as well as Dr. Eddie Y.K. NG, the Editor-in-Chief of JMIHI, for his kind guidance and support throughout the whole process of SI preparation.

\section{Guest Editors Biography}

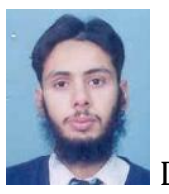
Dublin Institute of Technology, Dublin, Ireland since 2015. He did his PhD in CS from IIU, Pakistan in 2011. $\mathrm{He}$ is a Guest editor of Multimedia Tools and Applications, Telecommunication System Journal, and Information international Journal. He has published several papers at various international conferences and Journals. 
Dr. Adeel Javed has completed his BS-IT from CIIT, Lahore in 2006 and completed MS-CS from CIIT, Islamabad in 2008. Later in 2016, he completed his $\mathrm{PhD}$ in computer science from University of Otago, New Zealand. He served CIIT, Islamabad as a Lecturer from 2008 to 2010. He has also worked in King Saud University, Riyadh for a year in 2011. Now, he is working in University of Lahore in research and academic fields. 